\title{
Knockdown of CD146 reduces the migration and proliferation of human endothelial cells
}

\author{
Yanyong Kang ${ }^{1,3}$, Fengcai Wang ${ }^{1,2}$, Jing Feng ${ }^{1}$, Dongling Yang ${ }^{1}$, Xu Yang ${ }^{3}$, Xiyun Yan $^{1}$ \\ ${ }^{1}$ National Laboratory of Biomacromolecules, Institute of Biophysics, Chinese Academy of Science, Beijing 100101, China; ${ }^{2}$ Chemistry \\ Department, Anyang teacher's College, Anyang 455000, China: ${ }^{3}$ College of Life Science, Central China Normal University, Wuhan \\ 430079, China
}

Our previous study has demonstrated that CD146 molecule is a biomarker on vascular endothelium, which is involved in angiogenesis and tumor growth. However the mechanism behind is not clear. Here we have for the first time developed a novel CD146 blockade system using CD146 siRNA to study its function on endothelial cells. Our data showed that CD146 siRNA specifically blocked the expression of CD146 on both mRNA and protein levels, leading to the significant suppression of HUVEC proliferation, adhesion and migration. These results demonstrate that CD146 plays a key role in vascular endothelial cell activity and angiogenesis, and CD146 siRNA can be used as a new inhibitor for anti-angiogenesis therapy.

Cell Research (2006) 16:313-318. doi:10.1038/sj.cr.7310039; published online 16 March 2006

Keywords: small interfering RNA, adhesion molecule CD146, vascular endothelial cell

\section{Introduction}

Angiogenesis, the outgrowth of new capillaries from preexisting vessels, is essential in a variety of physiological and pathological processes, such as embryo implantation, female menstrual cycle, tumor, rheumatic and other diseases [1,2]. Numerous evidences have supported that the uncontrolled angiogenesis is a key point for tumor growth and metastasis [3,4], and thereby anti-angiogenesis has become one of the most promising strategies for cancer therapy [5]. Various specific bio-markers have been identified on tumor neovasculature, including VEGF [6], Tie2 receptor [7] and adhesion molecule integrin [8].

CD146, also named as MUC18, Mel-CAM, or MCAM, is a newly recognized cell adhesion molecule belonging to immunoglobulin superfamily [9-11]. It is initially identified as a progression marker of melanoma, and plays an

\footnotetext{
Correspondence: Xiyun Yan

Tel: +86-10-6488 8583; Fax: +86-10-6488 8584;

E-mail: yanxy@sun5.ibp.ac.cn

Received 12 Sep 2005; revised 1 Dec 2005; accepted 21 Dec 2005; published online 16 March 2006
}

important role in promoting melanoma progression and metastasis [10]. A fully humanized antibody against CD146, ABX-MA1, has been reported as an inhibitor to block the metastasis of human melanoma and disrupt blood vessel formation in vitro [12]. Recently, our study has shown that anti-CD146 monoclonal antibody AA98 inhibited angiogenesis and human tumor growth in xenografted mice [13]. However, the mechanism behind has not been elucidated. Here we develop a novel CD146 blockade system using CD146 siRNA to study the function of CD146 on endothelial cells.

RNA interference (RNAi), the process of sequencespecific post-transcriptional gene silencing, has become a powerful tool for the study of gene function [14-16]. It has been reported that a small interfering RNA (siRNA) targeting vascular endothelial growth factor could inhibit Ewing's sarcoma growth in a xenograft mouse model and ocular angiogenesis $[17,18]$. In this study, we for the first time use RNA interference technology to study the effect of CD146 siRNA on endothelial cell activity. Our data showed that CD146 siRNA specifically blocked the expression of CD146 on both mRNA and protein levels, leading to the significant suppression of HUVEC proliferation, adhesion 
and migration, suggesting that CD146 plays a key role in vascular endothelial cell activity and angiogenesis, and CD146 siRNA can be used as a new inhibitor for antiangiogenesis therapy.

\section{Materials and Methods}

\section{Preparation of siRNAs}

Three 21-nucleotide siRNAs were designed to target different coding sequences of human CD146 gene (Table 1), and synthesized by GeneChem (Shanghai, China). GFP siRNA was used as a control. By Blast searching, we found that the three CD146 siRNAs had no homology with any relevant human genes.

\section{Cell culture and transfection}

HUVECs, human vascular endothelial cells, were purchased from CellSystems (Biotechnologie Vertrieb $\mathrm{GmbH}$, Katharinen, Germany), and propagated in DMEM (Gibco, BRL) supplemented with $10 \%$ FCS, glutamine $(2 \mathrm{mM})$ and penicillin $(50 \mathrm{IU} / \mathrm{mL})$, streptomycin $(50 \mu \mathrm{g} / \mathrm{mL})$, and amphotericin $(1.25 \mu \mathrm{g} / \mathrm{mL})$. One day before transfection, the cells were seeded at a density of $2 \times 10^{5}$ cells/well onto 6-well-plate with antibiotics-free medium. When the cells were growing at $80 \%$ confluence, the siRNAs were transfected into the cells with Lipofectamine ${ }^{\mathrm{TM}} 2000$ reagent (Invitrogen, Carlsbad, CA) following the protocol provided by the manufacture.

\section{$R T-P C R$}

Total RNA was extracted from the transfected cells using RNeasy Mini Kit (Qiagen, Inc., Valencia, CA) as instructed. Reverse transcription was performed using oligo (dT) primers and Superscript II reverse transcriptase (Takara Shuzo, Japan) according to the manufacturer's instruction. A pair of primer, 5'- GAA GGA GGA GAA GAA CCG -3' (forward) and 5'- ACA GCC ACG ATG ACC AC $-3^{\prime}$ (reverse), was used to amplify the fragment of CD146 from $562 \mathrm{bp}$ to $1697 \mathrm{bp}$. GAPDH was used as an internal control with a forward primer 5'- ACC ACA GTC CAT GCC ATC AC -3', and a reverse primer 5'- TCC ACC ACC CTG TTG CTG TA -3'. Thirty cycles of PCR were performed as follows: $94{ }^{\circ} \mathrm{C}, 30 \mathrm{~s}, 52{ }^{\circ} \mathrm{C}, 60$ s, $72{ }^{\circ} \mathrm{C}, 90 \mathrm{~s}$. and then $72{ }^{\circ} \mathrm{C}, 10 \mathrm{~min}$. The RT-PCR products were examined by electrophoresis in $1 \%$ agarose gel containing $0.2 \mu \mathrm{g} / \mathrm{ml}$ ethidium bromide.

\section{SDS-PAGE and Western Blot}

For preparation of cell extracts, the 48 hour-transfected cells were washed thrice with ice-cold PBS, and lysed in lysis buffer (Tris-HCl $50 \mathrm{mM} \mathrm{pH}$ 8.0, NaCl $150 \mathrm{mM}$, EDTA $1 \mathrm{mM}$, Triton X-100 1\%, PMSF $100 \mu \mathrm{g} / \mathrm{ml}$ ) on ice for $20 \mathrm{~min}$. After centrifuging at $16000 \times \mathrm{g}$ for $2 \mathrm{~min}$ at $4^{\circ} \mathrm{C}$, the supernatants were analyzed by $10 \%$ SDS-PAGE and then electrophoretically transferred to nitrocellulose membrane (Invitrogen, Carlsbad, CA). After blocking $2 \mathrm{~h}$ with 5\% fat free milk at room temperature, the membranes were incubated with either anti-CD146 monoclonal antibody AA98 or anti-actin monoclonal antibody (Sigma, Deisenhofen, Germany), and then reacted with HRP-conjugated secondary antibody. Protein bands were visualized with enhanced chemiluminescence reagent (Pierce, Rockford, IL).

\section{Flow cytometry}

The trypsin-detached cells were processed to obtain single-cell suspension followed by staining with primary antibody AA98 in PBS with $1 \%$ BSA on ice for 40 min. After washing thrice in PBS with $1 \%$ BSA, cells were incubated with FITC-conjugated secondary antibody on ice for $30 \mathrm{~min}$, then washed and analyzed using a FACSCalibur flow cytometry system (Becton Dickinson, San Jose, CA). For cell apoptosis assay, the transfected cells were double-stained with FITC-conjugated Annexin V $(25 \mu \mathrm{g} / \mathrm{ml})$ and propidium iodide (PI, $50 \mu \mathrm{g} / \mathrm{ml}$ ) according to the manufacturer's protocol.

\section{MTT assay}

The 48-hour-transfected HUVECs in 96-well-plate were treated $4 \mathrm{~h}$ with $20 \mu \mathrm{l}$ of MTT dye $\left(5 \mathrm{mg} / \mathrm{ml}\right.$ in DMEM) at $37{ }^{\circ} \mathrm{C}$ and then incubated with $150 \mu 1$ of dimethyl sulfoxide (DMSO) for $5 \mathrm{~min}$. The color reaction was measured at $570 \mathrm{~nm}$ with a BioRad ELISA reader (Richmond, CA). The proliferation rate was calculated for each clone. Growth rate $(\%)=$ Absorbency of transfected cells/Absorbency of untransfected cells $\times 100 \%$. The mean and standard deviation of three relative proliferation rates for each clone were plotted.

\section{Cell adhesion assay}

The cells were first transfected with either CD146 siRNA or GFP siRNA, followed by culture for $48 \mathrm{~h}$, and then treated with $1 \times 10^{-4}$ M EDTA for $1 \mathrm{~min}$ at room temperature. The detached cells were washed with PBS, and then replaced into 6-wells culture plate at 2 $\times 10^{4}$ cells per well. After culture for $2 \mathrm{~h}$, the undetached cells were

Table 1 The sequences of three CD146 siRNAs and GFP siRNA

\begin{tabular}{|c|c|c|c|c|}
\hline siRNA & & mRNA(bp) & & sequences \\
\hline \multirow[t]{6}{*}{ CD146 } & No. 1 & $410-428$ & forward & 5'-CCA GCU CCG CGU CUA CAA AdTdT-3' \\
\hline & & & reverse & 5'-UUU GUA GAC GCG GAG CUG GdTdT-3' \\
\hline & No. 2 & $1009-1027$ & forward & 5'-UGA UAU CGC UGC UGA GUG AdTdT-3' \\
\hline & & & reverse & 5'-UCA CUC AGC AGC GAU AUC AdTdT-3' \\
\hline & No. 3 & 1828-1846 & forward & 5'-ACA UCG AUC UGA GGC AUU AdTdT-3' \\
\hline & & & reverse & 5'-UAA UGC CUC AGA UCG AUG UdTdT-3' \\
\hline \multirow[t]{2}{*}{ GFP siRNA } & & $1562-1580$ & forward & 5'-CUU CAG CCU CAG CUU GCC GdTdT-3' \\
\hline & & & reverse & 5'-CGG CAA GCU GAC CCU GAA GdTdT-3' \\
\hline
\end{tabular}




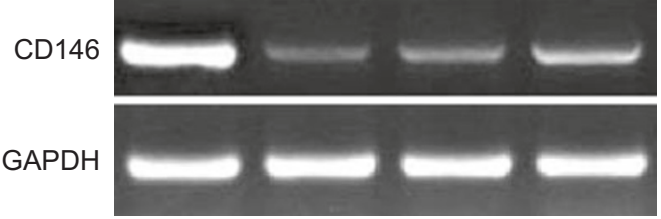

Figure 1 RT-PCR assay shows the mRNA level of CD146 and GAPDH in the HUVECs that treated with siRNAs. Lane 1, the cells treated with GFP siRNA. Lane 2-4, the cells treated with CD146 siRNA no. 1 , no. 2 and no. 3.

removed by washing with PBS and the attached cells were calculated under microscope. Three fields were viewed in each well and all the experiments were repeated three times independently.

\section{Wound healing assay}

HUVECs were cultured in a 6-well-culture-plate. For siRNA transfection, the cells were transfected using Lipofectamine 2000 transfection reagent as describe above. $24 \mathrm{~h}$ later, when cells reached $90 \%$ confluence, a single wound was created in the center of the cell monolayers by gentle removal of the attached cells with a sterile plastic pipette tip. The debris was removed by washing with serum free medium. After $24 \mathrm{~h}$ of incubation, the cells which migrated into the wounded area or protruded from the border of the wound were visualized and photographed under an inverted microscope. Each experiment was performed at least three times independently

\section{Statistical analysis}

The 2-tailed Student/test was used for statistical analysis. All data are presented as the mean \pm standard error of the mean (s.e.m.). A $P$-value of less than 0.05 was considered to be significant.

\section{Results}

CD146 siRNAs silence CD146 gene expression in HUVEC

To test whether CD146 siRNAs would down-regulate the expression of CD146 in HUVEC, we designed three CD146 siRNAs targeting different coding sequences of human CD146 gene (Table 1). After transfected with the siRNAs for $24 \mathrm{~h}$, the cells were lysed for analysis. RT-PCR assay showed that the three CD146 siRNAs all decreased the expression of CD146 at mRNA level, but they did not affect the expression of GAPDH in the same treated cells. In contrast, the control GFP siRNA did not affect both CD146 and GAPDH (Figure 1). These results indicate that the suppression of CD146 is specifically induced by CD146 siRNA. The CD146 siRNA No.1, which appeared the most effective among the three CD146 siRNAs, was selected for the following assays.

To test whether the CD146 expression was also inhibited by CD146 siRNA on the protein level, we performed Western blot and FACS analysis to detect the CD146 molecule

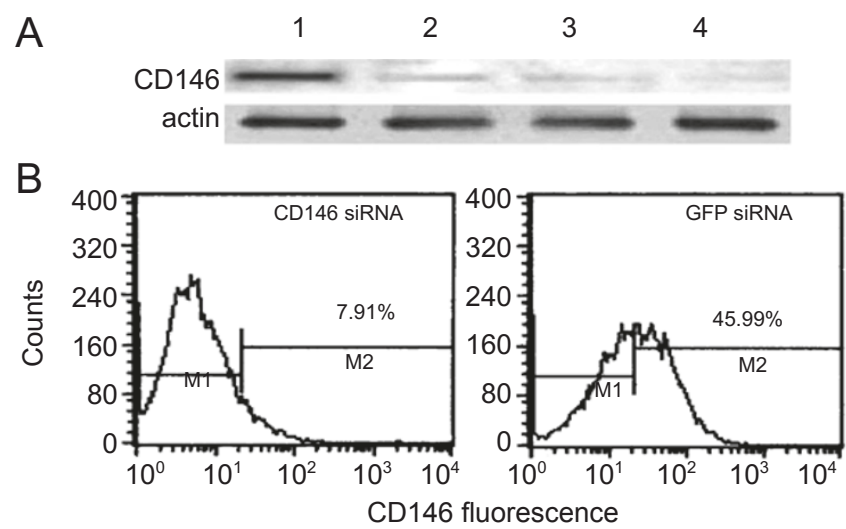

Figure 2 The CD146 expression is suppressed by CD146 siRNA. (A) Western Blot shows that CD146, rather than actin, was reduced in the HUVECs treated with CD146 siRNA. Lane 1. GFP siRNA-treated cells: Lane 2-4. Cells transected with CD146 siRNA in $20 \mathrm{nM}, 40 \mathrm{nM}$ and $80 \mathrm{nM}$. (B) FACS analysis shows the expression of CD146 in the cells treated with either CD146 siRNA or GFP siRNA.

in the treated cells. The results from Western blot (Figure 2A) showed that the expression of CD146, rather than actin, was dramatically reduced in the cells treated with CD146 siRNA, whereas the CD146 was not affected by GFP siRNA. Also, this reduction was found as in a dosedependent manner. These findings were further confirmed by FACS analysis (Figure 2B) that CD146 protein was specifically depleted in the cells after treated with CD146 siRNA. These results indicate that the CD146 siRNA can selectively block CD146 expression on both mRNA and protein level.

\section{Suppression of CD146 decreases cell adhesion}

To investigate the effect of CD146 siRNA on cell adhesion, we first treated the cells with either CD146 siRNA or GFP siRNA. Then the retreated cells were detached using EDTA treatment and re-cultured for another $2 \mathrm{~h}$. Afterwards the detached cells were washed off with PBS, and the adhered cells were visualized and calculated under microscope. As the results shown in Figure 3, the CD146 siRNA treated cells appeared tightly attached to the plate than those treated with GFP siRNA, suggesting that the CD146 siRNA suppressed the amount of CD146 molecule on cell surface, resulting in the decrease of cell adhesion.

\section{Suppression of CD146 decreases cell proliferation but not cell apoptosis \\ Then we examined the functional properties of CD146 in cell activities, including cell proliferation and apoptosis. By using MTT assay, we found that the CD146 siRNA inhibited (57\%) the proliferation of HUVEC cells, whereas}



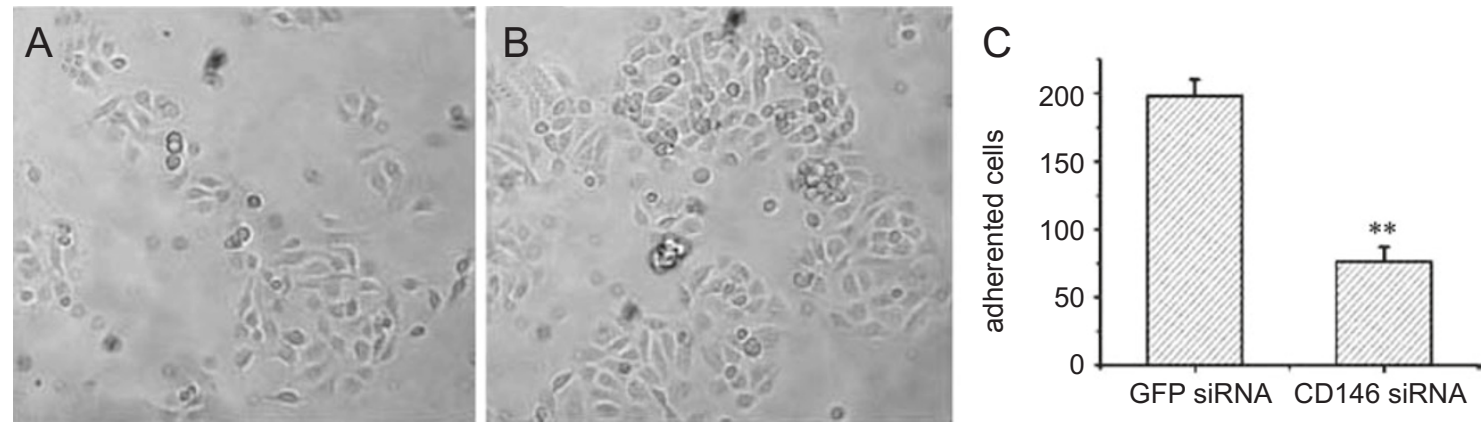

Figure 3 Cell adhesion is suppressed by CD146 siRNA. (A) Cells treated with CD146 siRNA, (B) Cells treated with GFP siRNA, (C) Quantity of the attached cells. The data present an average from three fields of view. Bars represent mean \pm S.D., asterisk, $P<$ 0.05 . Magnification $\times 100$

the control GFP siRNA did not affect cell growth (Figure 4A). These data suggest that the anti-proliferative effect of CD146 siRNA on HUVEC is specific and it is not due to the cytotoxic effect of siRNAs.

We further studied whether the inhibitory effect of CD146 siRNA on cell proliferation was due to its induction of cell apoptosis. FACS assay was performed by using FITC-conjugated Annexin V and PI as probes to detect apoptotic cells. It has been reported that during the early stage of apoptosis, phosphatidylserine is translocated from the inner cytoplasmic membrane to the outer surface, which could be specifically recognized by annexin $\mathrm{V}$ protein [19]. The results from FACS are presented in Figure 4, that no apoptotic cells were significantly detected in the HUVECs treated with either CD146 siRNA or GFP siRNA.

Suppression of CD146 decreases cell migration
We performed in vitro wound healing assay to assess the role of CD146 in cell migration. When cells reach $90 \%$ confluence, a single wound was created in the center of the cell monolayers by gentle removal of the attached cells with a sterile plastic pipette tip. The debris was removed by washing with serum free medium. Cell migration into the wound area was visualized under an inverted microscope at different time points. The results showed that the migration of CD146 siRNA treated-cells appeared significantly slower compared to the control GFP siRNA treated-cells (Figure 5).

\section{Discussion}

Angiogenesis is a complex process that is linked to the functional state of interendothelial junctions which are modulated by the state of proliferation and migration
A

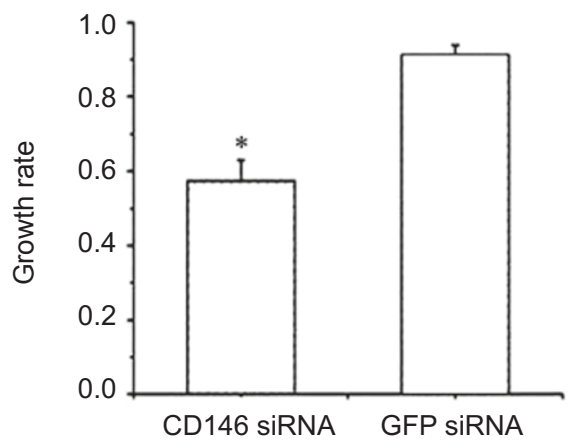

B

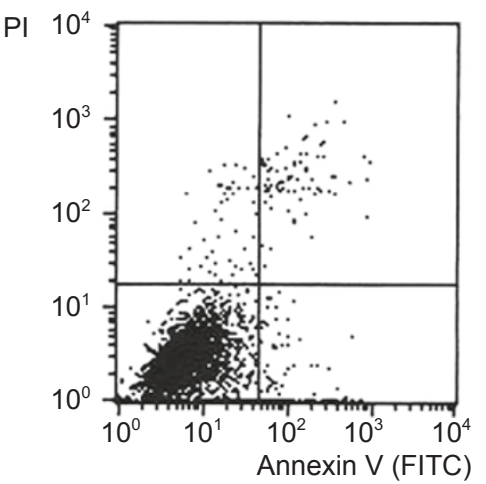

C

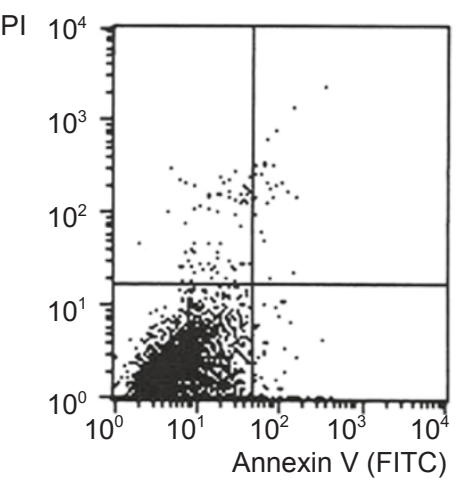

Figure 4 HUVEC proliferation, rather than cell apoptosis, is inhibited by CD146 siRNA. (A) MTT assay shows that CD146 siRNA suppresses cell proliferation comparing with GFP siRNA. Data are mean \pm S.D. $(n=3)$. $* P<0.05$ compared with GFP siRNA treated cells. The cells treated with either CD146 siRNA (B) or GFP siRNA (C) did not show any effect on cell apoptosis, as determined by FACS analysis with both PI and FITC-conjugated Annexin V. 

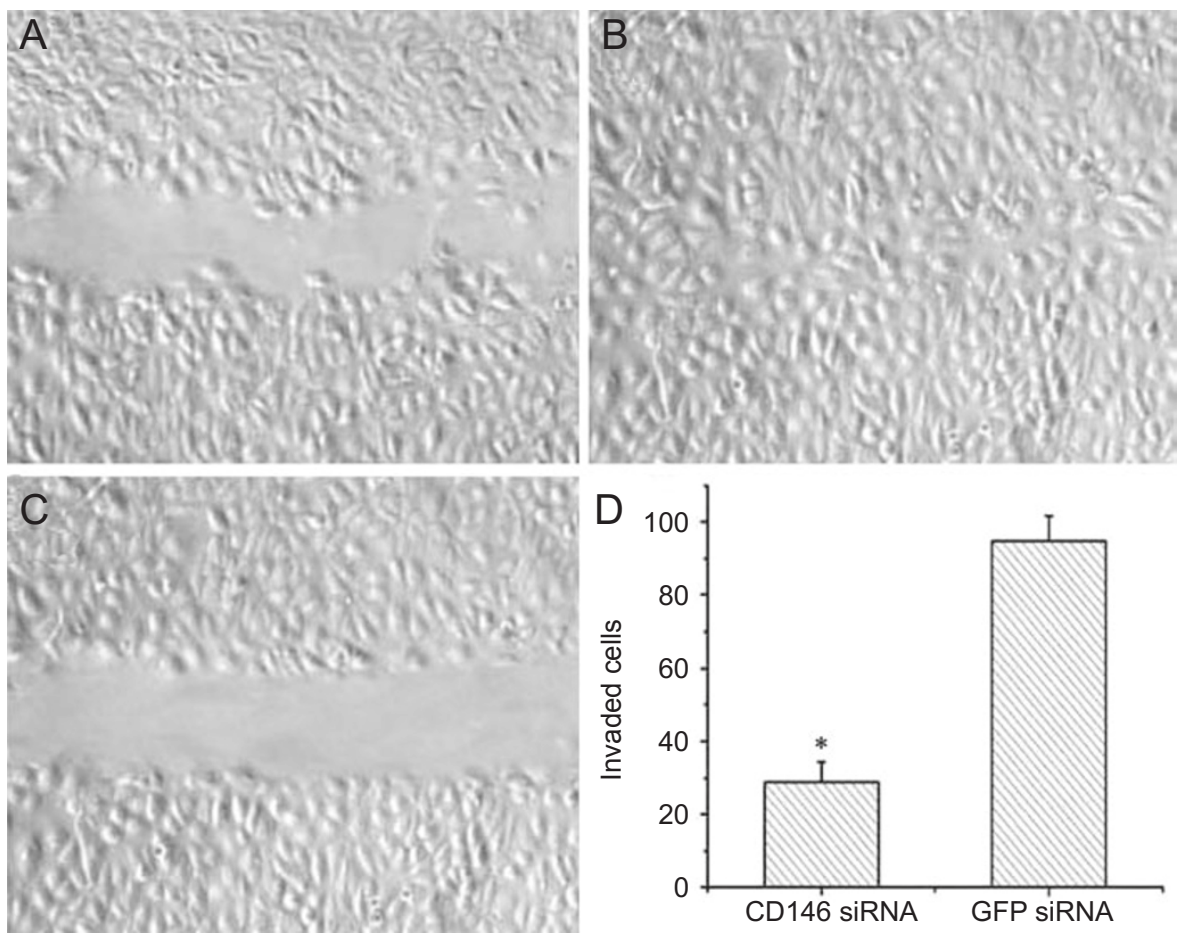

Figure 5 Wound healing assay shows that the CD146 siRNA inhibits cell migration. Cells were first treated with either CD146 siRNA (A) or GFP siRNA (B). When the cells grew up to $90 \%$ confluence, a single wound was made in the center of cell monolayer and cell debris was removed by washing (C). After $24 \mathrm{~h}$ of incubation, the wound closure areas were visualized under an inverted microscope with a magnification $\times 100$, and the migrated cells were counted. (D) Quantity of migrated cells presents an average from three experiments independently. Statistical analysis, $* P<0.05$.

of endothelial cells. In our previous study, using an antiCD146 antibody as a function-blocking agent, we found that CD146 was selectively localized in tumor neovasculatures and involved in angiogenesis and tumor growth. However, the mechanism of CD146 mediated angiogenesis is unknown. Here we developed a novel CD146 blockade system to study the function of CD146 in vascular endothelial cells, and to better understand the mechanism of CD146 participation in angiogenesis in vivo.

The activities of endothelial cell including cell adhesion, proliferation and migration are critical process for angiogenesis [20]. Here we found, for the first time, that CD146 siRNA blocked the expression of CD146 on both mRNA and protein levels, leading to significant suppression of HUVEC cell proliferation, adhesion and migration but not apoptosis. These present results are consistent with the previous data using an antibody-based approach [13], and further demonstrate that CD146 plays an important role in the adhesion, migration and proliferation of HUVEC, which are critical processes in angiogenesis.

The involvement of CD146 in controlling cell migration has been reported previously by two different antibodybased approaches. Solvery et al. had shown that bead im- mobilized anti-CD146 antibody, $\mathrm{P} 1 \mathrm{H} 12$, can inhibit in vitro migration of HUVECs in "wound closure" experiments [21]. Our previous data had also shown that the anti-CD146 antibody, AA98, can inhibit in vitro migration of HUVECs in a modified Boyden chamber assay [13]. It is hard for both experiments to answer whether the anti-CD146 antibodies act as an agonist or antagonist in cell activity. In this study, we provide direct evidences that the knockdown of CD146 expression by siRNA decreases HUVEC cell mobility, including cell proliferation, adhesion and migration. These data also provide the explanation that the anti-CD146 mAb AA98 inhibited angiogenesis through blocking the function of CD146 on endothelial cells. It will be interesting to further determine whether CD146 functionally connects to structural and signaling networks that control migration, proliferation and endothelial monolayer formation.

In the process of angiogenesis, endothelial cells migrate into the perivascular space, proliferate there and adhere to one another and to the extracellular matrix to form new capillaries. This process is regulated by endothelial adhesion molecules and extracellular matrix molecules [22]. CD146 is a member of immunoglobulin family that is specifically expressed in the endothelial cells, localized to the cell-cell 
junction. Our results showed that down-regulated expression of CD146 inhibited the adhesive activity of endothelial cells, which confirmed that CD146 is very important for the adhesion of endothelial in angiogenesis. A recent study showed that blockade of CD146 with a neutralizing antibody could inhibit the expression of MMP-2 and attachment of metastatic melanoma cells to HUVECs.

Taking these studies into account, we suggest that CD146 is a key molecule in vascular endothelial cell activity and angiogenesis, and the CD146 siRNAs, which suppress endothelial cell adhesion, migration and proliferation, are a new tool for studying the function of CD146 in endothelial cells, and it will be applied as a new inhibitor for anti- angiogenesis therapy.

\section{Acknowledgements}

This work was supported by the National Natural Science Foundation of China (90406020), CAS Grant (KSCXZ-SW-213) and Hi-Tech Research and Development Program of China (863 Program, 2003AA215061).

\section{References}

1 Carmeliet P. Angiogenesis in health and disease. Nat Med 2003; 9:653-660.

2 Folkman J. Angiogenesis in cancer, vascular, rheumatoid and other diseases. Nat Med 1995; 1:27-31

3 Carmeliet P, Jain RK. Angiogenesis in cancer and other diseases. Nature 2000; 14:249-257.

4 Zetter BR. Angiogenesis and tumor metastasis. Annu Rev Med 1998; 49:407-424.

5 Nelson NJ. Angiogenesis research is on fast forward. J Natl Cancer Inst 1999; 91:820-822.

6 Ferrara N, Hillan KJ, Gerber HP, et al. Discovery and development of bevacizumab, an anti-VEGF antibody for treating cancer. Nat Rev Drug Discov 2004; 3:391-400.

7 Tournaire R, Simon MP, le Noble F, et al. A short synthetic peptide inhibits signal transduction, migration and angiogenesis mediated by Tie2 receptor. EMBO Rep 2004; 5:262-267.

8 Brooks, PC, Clark RA, Cheresh DA. Requirement of vascular integrin alpha $v$ beta 3 for angiogenesis. Science 1994; 264:569571.
9 Lehmann JM, Riethmuler G, Johnson JP. MUC18, a marker of tumor progression in human melanoma, shows sequence similarity to the neural cell adhesion molecules of the immunoglobulin superfamily. Proc Natl Acad Sci U S A. 1989; 86:9891-9895.

10 Shih IM, Elder DE, Speicher D, et al. Isolation and functional characterization of the A32 melanoma associated antigens. Cancer Res. 1994; 54:2514-2520.

11 Bardin N, Frances V, Lesaule G, et al. Identification of the SEndo1 endothelial-associated antigen. Biochem Biophys Res Commun. 1996; 218:210-216.

12 Mills L, Tellez C, Huang S, et al. Fully Human Antibodies to MCAM/MUC18 inhibit tumor growth and metastasis of human melanoma. Cancer Res 2002; 62:5106-5114.

13 Yan X, Lin Y, Yang D, et al. A novel anti-CD146 monoclonal antibody, AA98, inhibits angiogenesis and tumor growth. Blood 2003; 102:184-191.

14 Elbashir SM, Harborth J, Lendeckel W, et al. Duplexes of 21nucleotide RNAs mediate RNA interference in cultured mammalian cells. Nature 2001; 411:494-498.

15 Caplen NJ, Parrish S, Imani F. Specific inhibition of gene expression by small double-stranded RNAs in invertebrate and vertebrate systems. Proc Natl Acad Sci U S A 2001; 98:9742-9747.

16 Fire A, Xu S, Montgomery MK, et al. Potent and specific genetic interference by double-stranded RNA in caenorhabditis elegans. Nature 1998; 391:806-811.

17 Guan H, Zhou Z, Wang H, et al. A small interfering RNA targeting vascular endothelial growth factor inhibits Ewing's sarcoma growth in a xenograft mouse model. Clin Cancer Res 2005; 11:2662-2669.

18 Kim B, Tang Q, Biswas PS, et al. Inhibition of ocular angiogenesis by siRNA targeting vascular endothelial growth factorpathway genes; therapeutic strategy for herpetic stromal keratitis. Am J Pathol. 2004; 165:2177-2185

19 Zhang G, Gurtu V, Kain SR, et al. Early detection of apoptosis using a fluorescent conjugate of annexin V. Biotechniques 1997; 23:525-531.

20 Folkman J. Tumor angiogenesis: therapeutic implications. N Engl J Med 1971; 285:1182-1186.

21 Solovey AN, Gui L, Chang L, et al. Identification and function assessment of endothelial P1H12. J Lab Clin Med 2001; 138:322331.

22 Joyce B. Cell adhesion and angiogenesis. J Clin Invest 1997; 99:373-376

Edited by Zhong Cheng Zheng 